\title{
Ionic Liquids: Potential Electrolytes for Electrochemical Applications
}

\author{
S. Zein El Abedin, ${ }^{1,2}$ K. S. Ryder, ${ }^{3}$ O. Höfft, ${ }^{2}$ and H. K. Farag ${ }^{4}$ \\ ${ }^{1}$ Electrochemistry and Corrosion Laboratory, National Research Centre, Dokki, Cairo 12622, Egypt \\ ${ }^{2}$ Institute of Particle Technology, Clausthal University of Technology, Arnold-Sommerfeld-Street 6, \\ 38678 Clausthal-Zellerfeld, Germany \\ ${ }^{3}$ Department of Chemistry, University of Leicester, Leicester LE1 7RH, UK \\ ${ }^{4}$ Inorganic Chemistry Department, National Research Centre, Dokki, Cairo 12622, Egypt
}

Correspondence should be addressed to S. Zein El Abedin, sherif.zein@tu-clausthal.de

Received 12 August 2012; Accepted 12 August 2012

Copyright (C) 2012 S. Zein El Abedin et al. This is an open access article distributed under the Creative Commons Attribution License, which permits unrestricted use, distribution, and reproduction in any medium, provided the original work is properly cited.

In recent years, ionic liquids (ILs) have received great interest since they have unconventional physical properties superior, in many respects, to those of common molecular liquids. They are usually nonvolatile, nonflammable, less toxic, and are good solvents for both organics and inorganics. They have extremely large electrochemical windows, up to seven volts and high thermal stability, up to $300-400^{\circ} \mathrm{C}$. Most of the liquid known hitherto have very low vapor pressure (e.g., at or near room temperature around $10^{-11}-10^{-10} \mathrm{mbar}$ ) which makes new experiments under vacuum conditions possible. Low vapor pressure also reduces solvent losses in bulk processing and so ILs are the subject of much interest and speculation in respect of green chemistry. Moreover, the ionic liquids are "designable" as it is possible to design ILs with the necessary properties for specific applications by a proper choice of cations and anions. In addition to their extraordinary features, ionic liquids also typically have reasonable conductivities and viscosities contributing to their potential as neoteric solvents for a wide variety of applications including electrodeposition, electrocatalysis, electropolymerization, electrochemical capacitors, sensors, separations, and organic synthesis. They are also being developed for energy devices such as batteries, fuel cells, and solar photoelectrochemical cells. Furthermore, they are being explored for possible applications in the nuclear fuel cycle. The present themed issue demonstrates the potentialities of ionic liquids in electrochemical applications.
The "Ionic Liquids" themed issue contains some interesting papers exploring the high potential of ionic liquids as electrochemical solvents. As the electrochemical stability of ionic liquids is an important characteristic for their applications as electrolytes, quantum chemical calculations are employed to predict the electrochemical stability of ionic liquids. It is shown in this issue that the quantum chemical calculation is an excellent approach to predict the electrochemical window of ionic liquids. A comprehensive overview on the electrochemical applications of ionic liquids in spent fuel reprocessing and nuclear waste management is presented. The current status of the research on the electrochemical recovery of actinides and fission products using ionic liquids is systematically described.

The importance of ionic liquids for the synthesis of conducting polymers and nanoparticles is highlighted in another review paper with the focus on the electrochemical sensors and biosensors based on ionic- liquid-/compositemodified electrodes. The modification of a typical cationexchange polymeric membrane by inclusion of the IL cation into the matrix structure and its effect on chemical, thermal, and charge transport is also presented in this issue. The ionic-liquid-modified membranes are interesting for a wide variety of applications, particularly for electrochemical devices. The electrodeposition of magnesium from different imidazolium-based ionic liquids is also described. The electrodeposition of $\mathrm{Mg}$ is a good example for the prospects 
of ionic liquids in the electrodeposition of reactive metals which in the past were only accessible using high temperature molten salts or, in part, organic solvents.

S. Zein El Abedin

K. S. Ryder

O. Höfft

H. K. Farag 


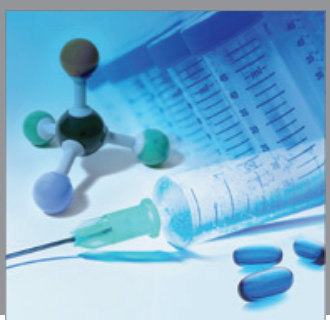

International Journal of

Medicinal Chemistry

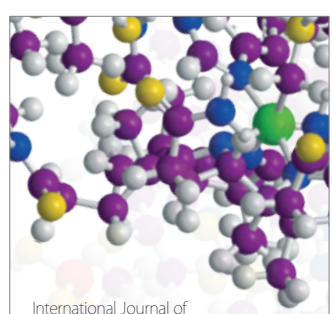

Carbohydrate Chemistry

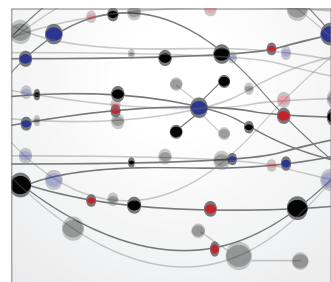

The Scientific World Journal
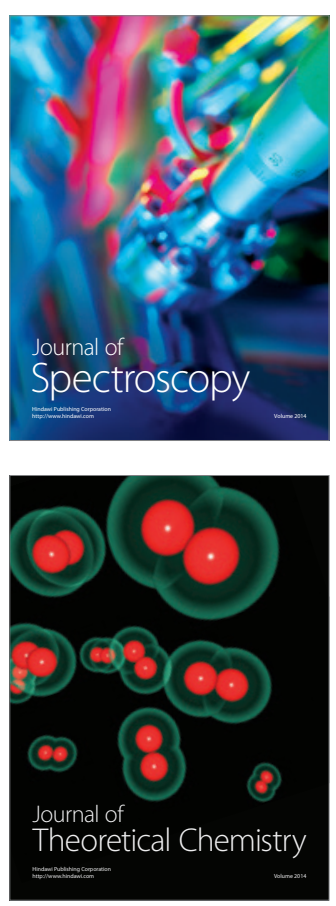
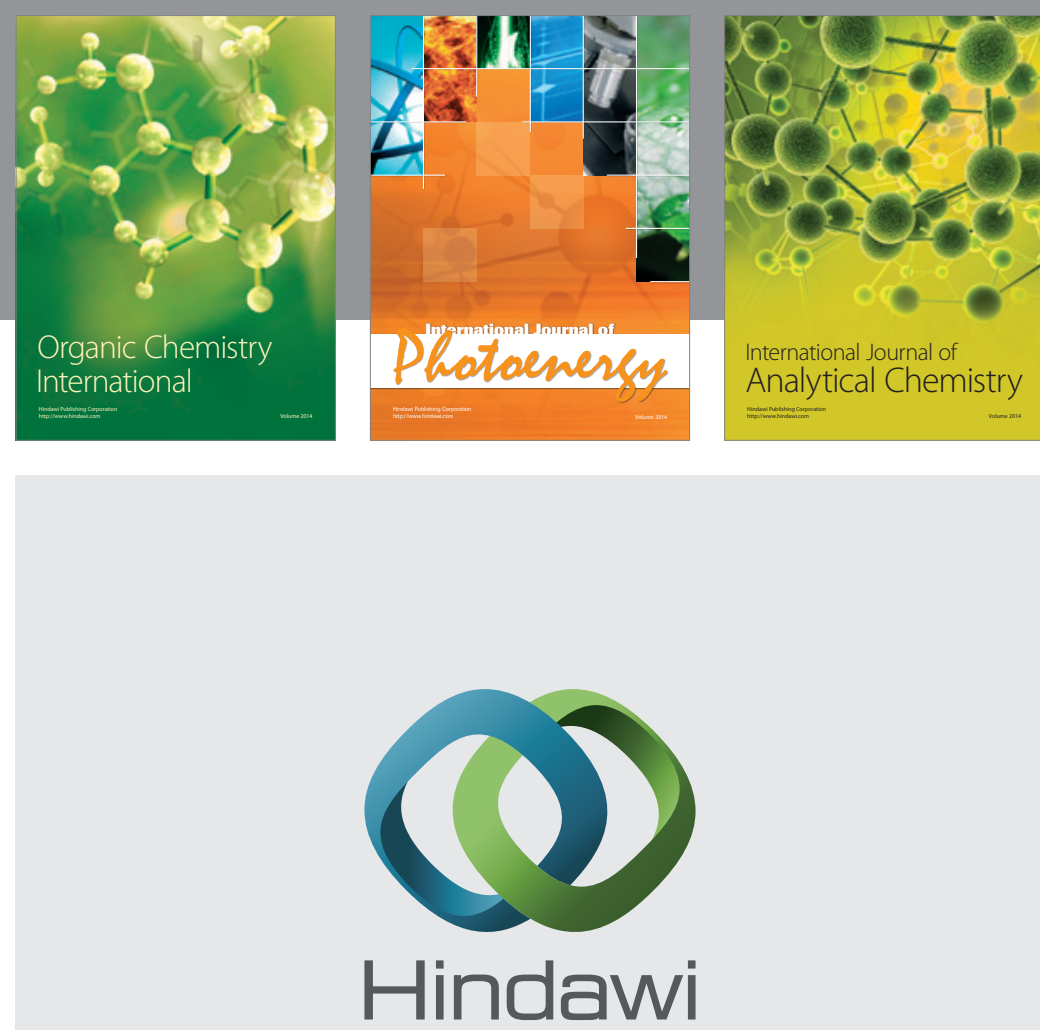

Submit your manuscripts at

http://www.hindawi.com
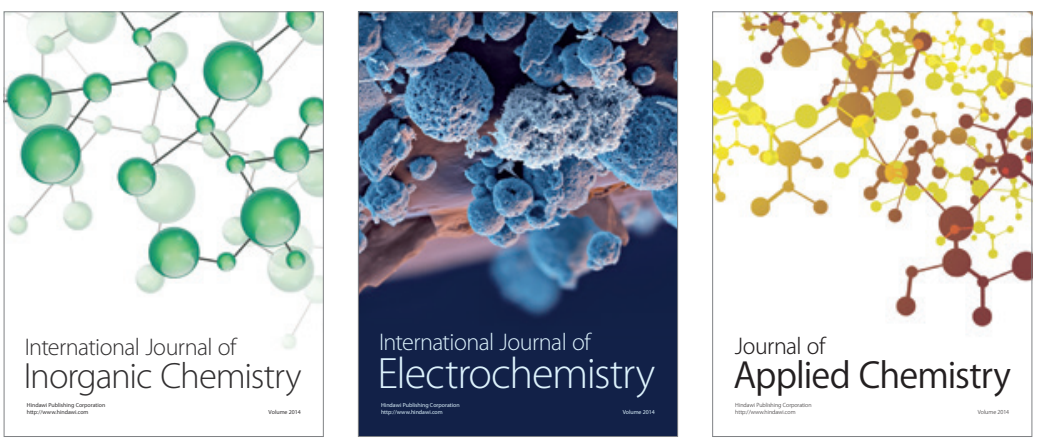

Journal of

Applied Chemistry
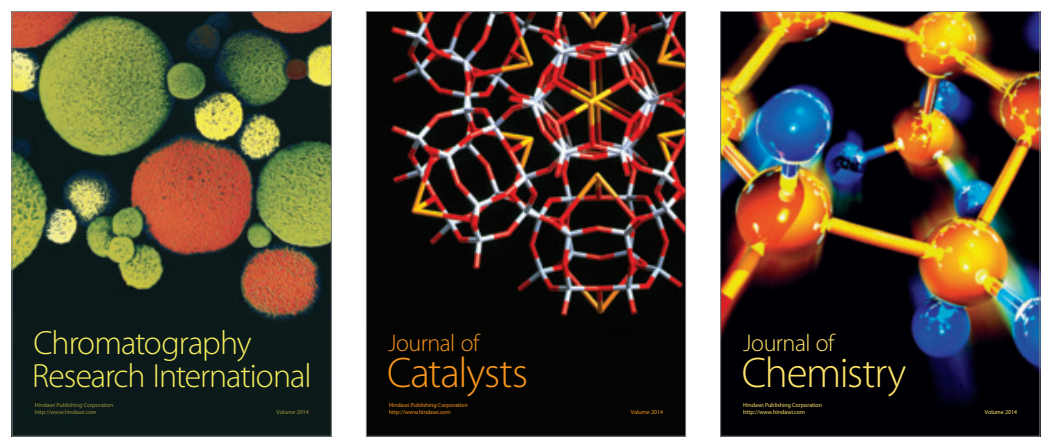
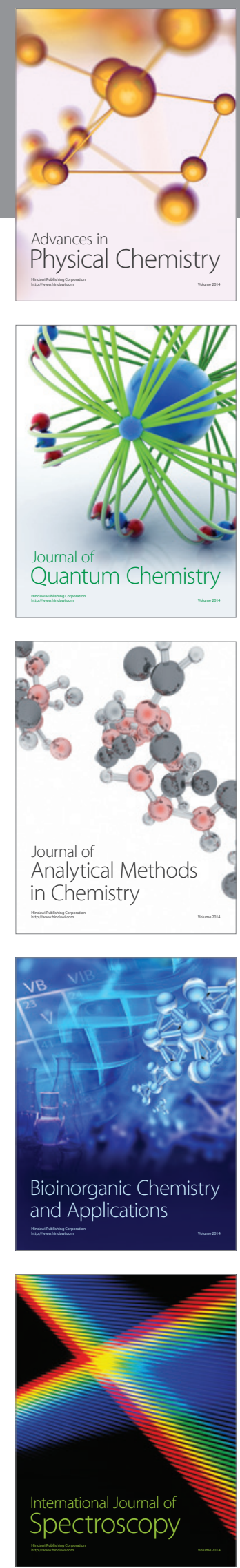